\title{
ARTICLE
}

Molecular Diagnostics

\section{Intratumoural expression of deoxycytidylate deaminase or ribonuceotide reductase subunit M1 expression are not related to survival in patients with resected pancreatic cancer given adjuvant chemotherapy}

\author{
N. O. Elander ${ }^{1}$, K. Aughton ${ }^{1}$, P. Ghaneh ${ }^{1}$, J. P. Neoptolemos ${ }^{1}$, D. H. Palmer ${ }^{1}$, T. F. Cox ${ }^{1}$, F. Campbell ${ }^{1}$, E. Costello ${ }^{1}$, C. M. Halloran ${ }^{1}$, \\ J. R. Mackey ${ }^{2}$, A. G. Scarfe ${ }^{2}$, J. W. Valle $\mathbb{D}^{3}$, A. C. McDonald ${ }^{4}$, R. Carter ${ }^{5}$, N. C. Tebbutt ${ }^{6}$, D. Goldstein ${ }^{7}$, J. Shannon ${ }^{8}$, C. Dervenis ${ }^{9}$, \\ B. Glimelius ${ }^{10}$, M. Deakin ${ }^{11}$, R. M. Charnley ${ }^{12}$, A. Anthoney ${ }^{13}$, M. M. Lerch ${ }^{14}$, J. Mayerle ${ }^{15}$, A. Oláh ${ }^{16}$, M. W. Büchler $^{17}$ and \\ W. Greenhalf ${ }^{1}$ for the European Study Group for Pancreatic Cancer
}

BACKGROUND: Deoxycytidylate deaminase (DCTD) and ribonucleotide reductase subunit M1 (RRM1) are potential prognostic and predictive biomarkers for pyrimidine-based chemotherapy in pancreatic adenocarcinoma.

METHODS: Immunohistochemical staining of DCTD and RRM1 was performed on tissue microarrays representing tumour samples from 303 patients in European Study Group for Pancreatic Cancer (ESPAC)-randomised adjuvant trials following pancreatic resection, 272 of whom had received gemcitabine or 5-fluorouracil with folinic acid in ESPAC-3(v2), and 31 patients from the combined ESPAC-3(v1) and ESPAC-1 post-operative pure observational groups.

RESULTS: Neither log-rank testing on dichotomised strata or Cox proportional hazard regression showed any relationship of DCTD or RRM1 expression levels to survival overall or by treatment group.

CONCLUSIONS: Expression of either DCTD or RRM1 was not prognostic or predictive in patients with pancreatic adenocarcinoma who had had post-operative chemotherapy with either gemcitabine or 5-fluorouracil with folinic acid.

British Journal of Cancer (2018) 118:1084-1088; https://doi.org/10.1038/s41416-018-0005-1

\section{INTRODUCTION}

Ductal adenocarcinoma of the pancreas is among the five leading causes of cancer-related death worldwide. ${ }^{1,2}$ Post-operative chemotherapy with pyrimidine monotherapy or combination regimens is now the standard of care following resection. ${ }^{3-9}$ Biomarkers that could select patients for specific types of chemotherapy to improve survival even further would be of significant clinical value in this disease.

The biological response to 5-fluorouracil and gemcitabine is regulated by a series of proteins involved in the transmembrane uptake and intracellular metabolism of pyrimidines, and several of these are potential biomarkers for pyrimidine-based chemotherapy. ${ }^{2,10}$ Recently, we have reported that high expression of human equilibrative nucleotide transporter (hENT)- 1 was associated with improved overall survival in patients randomised to gemcitabine in the ESPAC-3(v2) adjuvant trial, but not in those who had received 5 -fluorouracil with folinic acid. ${ }^{11}$

Deoxycytidylate deaminase (DCTD) converts phosphorylated gemcitabine into its inactive metabolite ${ }^{12}$ and ribonucleotide reductase subunit 1 (RRM1) is a key target of the bioactive gemcitabine metabolite. ${ }^{13}$

In the present study, we assessed whether intratumoural expression of DCTD or RRM1 may be prognostic for patients with pancreatic adenocarcinoma who had had post-operative adjuvant chemotherapy with either gemcitabine or 5-fluoruracil with folinic acid in the European Study Group for Pancreatic Cancer (ESPAC)-3 (v2)-randomised adjuvant trial, ${ }^{4}$ and in patients from the combined ESPAC-1 and ESPAC-3(v1) post-operative pure observational groups. ${ }^{14}$

\footnotetext{
${ }^{1}$ Cancer Research U.K. Liverpool Cancer Trials Unit, University of Liverpool, Liverpool, UK; ${ }^{2}$ Cross Cancer Institute and University of Alberta, Edmonton, Canada; ${ }^{3}$ University of Manchester/The Christie NHS Foundation Trust, Manchester, UK; ${ }^{4}$ The Beatson West of Scotland Cancer Centre, Glasgow, UK; ${ }^{5}$ Glasgow Royal Infirmary, Glasgow, UK; ${ }^{6}$ Austin Health, Melbourne, VIC, Australia; ${ }^{7}$ Prince of Wales hospital and Clinical School, University of New South Wales, Sydney, NSW, Australia; ${ }^{8}$ Nepean Cancer Centre and University of Sydney, Camperdown, NSW, Australia; ${ }^{9}$ The Agia Olga Hospital, Athens, Greece; ${ }^{10}$ Department of Immunology, Genetics and Pathology, Uppsala University, Uppsala, Sweden; ${ }^{11}$ University Hospital, North Staffordshire, Staffordshire, UK; ${ }^{12}$ Freeman Hospital, Newcastle upon Tyne, UK; ${ }^{13}$ St James's University Hospital, Leeds, UK; ${ }^{14}$ Department of Medicine A, University Medicine Greifswald, Greifswald, Germany; ${ }^{15}$ Department of Medicine II, University Hospital of the Ludwig-Maximilians-University Munich, Munich, Germany; ${ }^{16}$ The Petz Aladar Hospital, Gyor, Hungary and ${ }^{17}$ Department of Surgery, University of Heidelberg, Heidelberg, Germany Correspondence: W Greenhalf (greenhaf@liv.ac.uk)
} 
Table 1. Summary of numbers and outcomes in the respective arms of the original trials

\begin{tabular}{lllll}
\hline Trial & Arm & $n$ & mOS $(95 \% \mathrm{Cl})$ & Reference \\
\hline ESPAC- 3(v2) & GEM & 537 & $23.6(21.4-26.4)$ & 4 \\
ESPAC-3(v2) & 5FU & 551 & $23.0(21.1-25.0)$ & \\
Pooled ESPAC-1/ESPAC-3(v1) & OBS & 225 & $16.8(14.3-19.2)$ & 14 \\
\hline
\end{tabular}

GEM gemcitabine, 5FU 5-fluorouracil with folinic acid, $O B S$ observational arm, mOS median overall survival, 95\% Cl 95\% confidence interval

\section{MATERIALS AND METHODS}

Study design and tissue microarray manufacture

The translational ESPAC-T studies received ethical committee approval for characterisation of tumour markers for chemotherapy from the Liverpool (Adult) Research Ethics Committee (07/H1005/ 87). The design of the ESPAC-1 and ESPAC-3(v2) trials, and the generation of tissue microarrays (TMAs), have been described previously. $3,4,6,11,14$

Immunohistochemistry on tissue microarray sections The primary antibodies were validated in accordance with the principles stated by the ESPAC Steering Committee

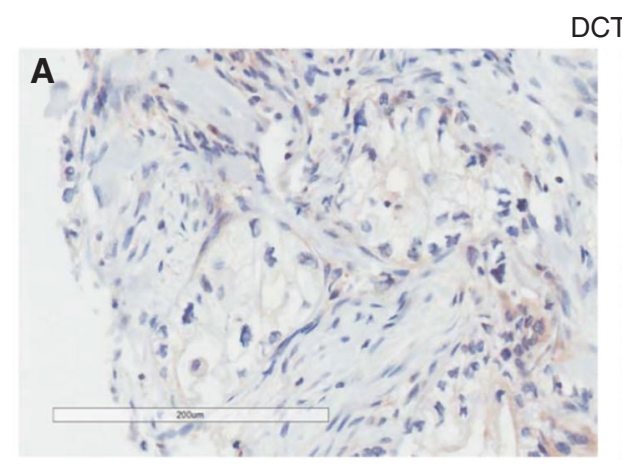

DCTD
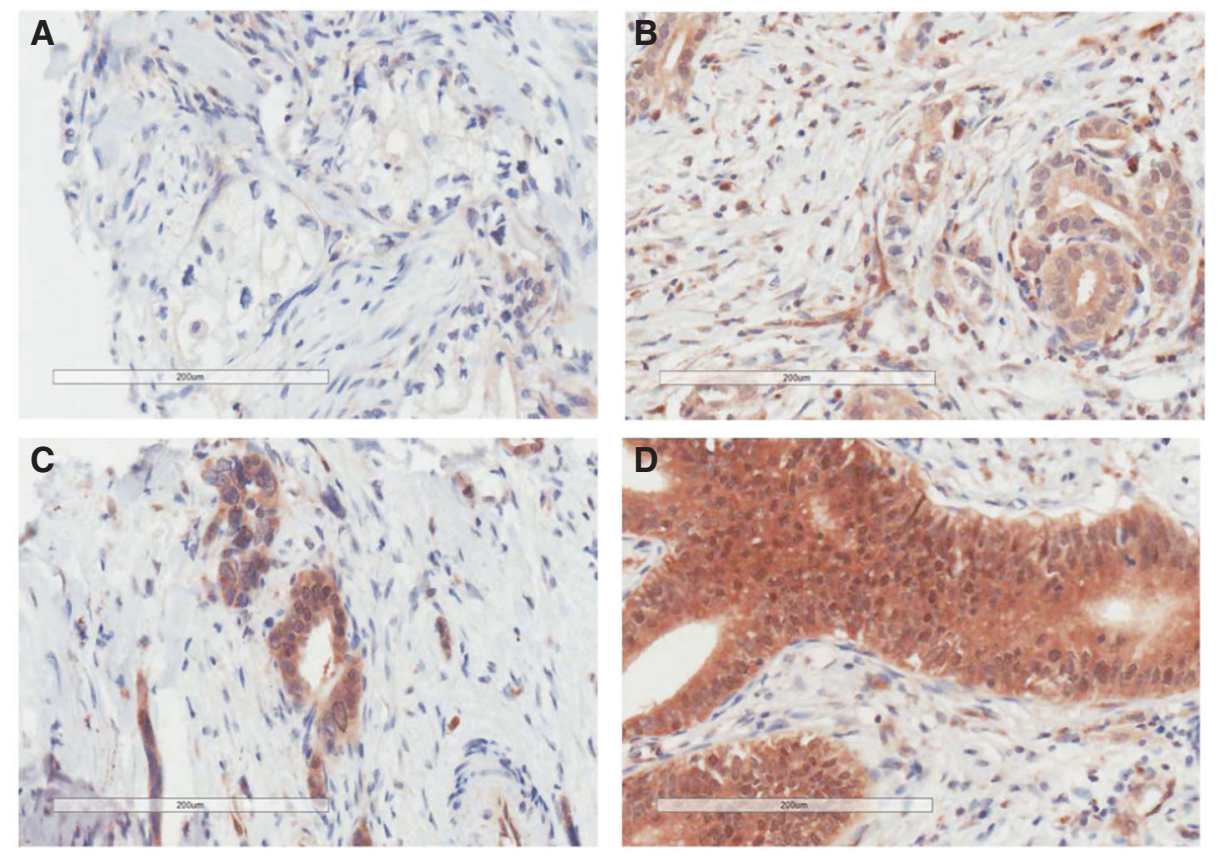

RRM1
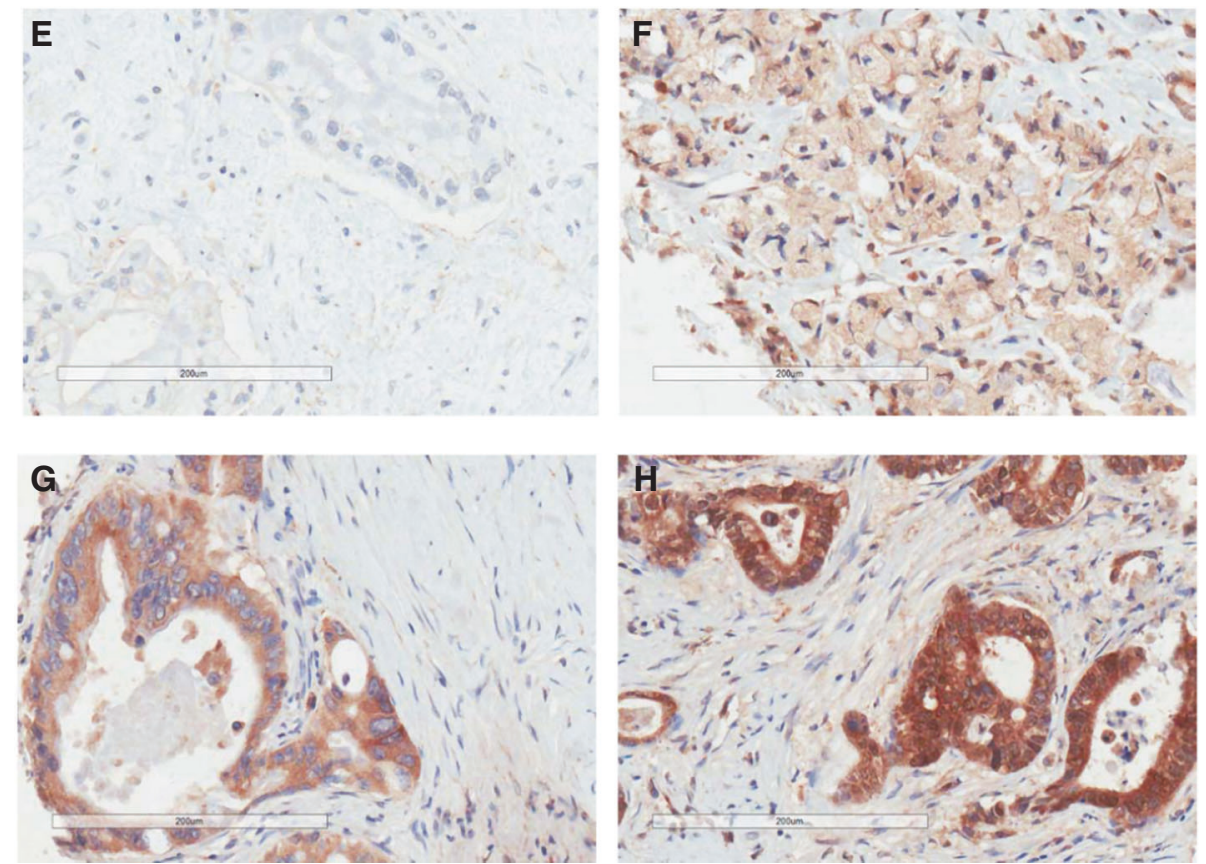

Fig. 1 DCTD and RRM1 immunhistochemistry scoring. Representative images of DCTD negative (a), weak (b), moderate (c), and strong (d) expressing tumours, and RRM1 negative (e), weak (f), moderate $(\mathbf{g})$ and strong $(\mathbf{h})$ expressing tumours, respectively 
A

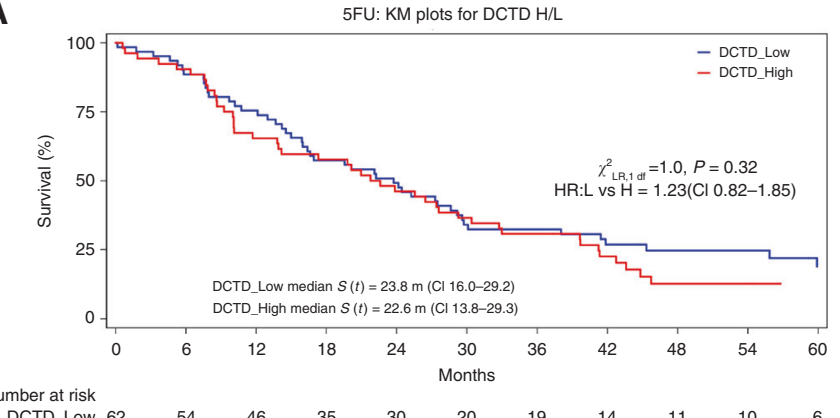

B

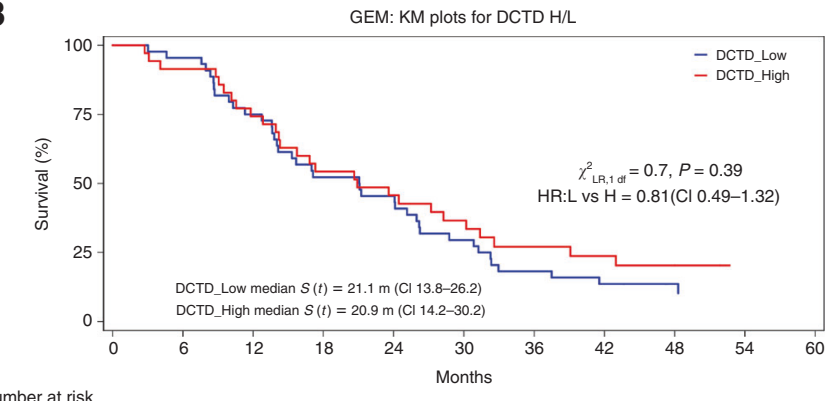

D

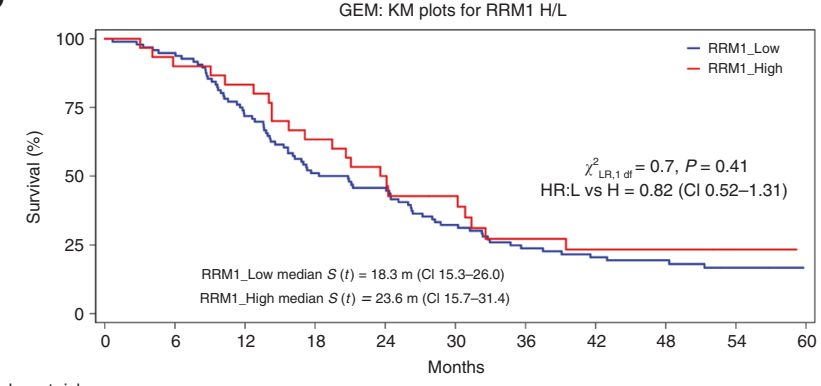

Number at risk

$\begin{array}{rllllllllll}\text { Rumber at risk } & & & & & & & & \\ \text { RRM1_Low 96 } & 91 & 69 & 49 & 44 & 31 & 22 & 19 & 15 & 10 & 8 \\ \text { RRM1_High 30 } & 27 & 25 & 19 & 15 & 11 & 7 & 6 & 6 & 5 & 3\end{array}$

Fig. 2 Kaplan-Meier survival curves of patient strata dichotomised on DCTD (a, b) and RRM1 (c, d) expression status (negative/weak $=0 / 1$, moderate/strong $=2 / 3$ ). 5-FU patients treated with 5-fluorouracil and folinic acid, GEM patients treated with gemcitabine, $y$ axis proportion of patients being alive, xaxis weeks from randomisation. $p$ values for log-rank $x^{2}$ analyses are given in the respective graph

(Supplementary Materials and Methods, antibodies were validated according to Good Clinical Practice Guidelines as shown in supplementary figures 1 to 4). TMA blocks of core biopsies were cut in $3 \mu \mathrm{m}$ sections and placed on Superfrost Ultra Plus ${ }^{\oplus}$ slides (Thermo Fisher Scientific Inc., Waltham, MA, USA). Deparaffinisation and antigen retrieval were performed with the PT-Link (Dako, Glostrup, Denmark) system and the $\mathrm{pH} 9.0$ target retrieval buffer. All buffers and reagents were provided in the EnVision ${ }^{\text {TM }}$ kit (Dako): slides were washed in Tris-buffered saline with $0.05 \%$ Tween-20 (TBS-T) before treated with peroxidase blocker for $10 \mathrm{~min}$. Following TBS-T washes, samples were incubated with primary antibody diluted 1:200 (anti-DCTD, 60 min incubation time) or 1:50 (anti-RRM1, 30 min incubation time), followed by secondary Horse Radish Peroxidase Horse Radish Peroxidase (HRP)-conjugated antibody, repeated TBS-T washes, and diamensobenzidine according to supplier's recommendation. Slides were washed in TBS-T and distilled water and counterstained in Hematoxylin Gills III and dehydrated via a series of ethanol gradients and fresh Xylen, before being mounted under cover glasses.

\section{Scoring}

The tumour cell compartments of all samples were scored by one experienced pancreas pathologist (F.C.) and one trained assistant (N.O.E.) according to a $0-3$ system ( $0=$ no staining, 1 = weak, 2 =moderate, $3=$ strong staining) both being blinded to patient identity and clinical data. If staining intensity within the core was not consistent, the most commonly observed pattern was scored. Any disagreement was resolved through discussion and a consensus decision. Each patient was given a single scoring grade equal to the mean over cores, rounded to the nearest integer. Antibodies were validated according to Good Clinical Practice Guidelines as shown in supplementary figures 1 to 4 where the supplementary material is referenced.
Statistics

Survival from date of randomisation was analysed using Kaplan-Meier curves, with differences between groups assessed using the log-rank test. Univariate and multivariate analyses were carried out using Cox proportional hazards. Presuming a symmetric 0.5:0.5 ratio between 'high' vs. 'low' would mean that a total of 66 events are required to detect a Hazard Ratio (HR) of 2.0 with 0.05 statistical significance level and an $80 \%$ power. All analyses were carried out using SAS version 9.3 software (SAS Institute, Cary, NC).

\section{RESULTS}

Patients and scoring of DCTD and RRM1

In total, 303 patients had provided written informed consent for use of their tissue for research and had tissue available for immunohistochemical staining, of whom 272 had had chemotherapy in the ESPAC3(v2)-randomised adjuvant trial, ${ }^{4}$ and 31 had pure observation following resection from the combined ESPAC-1/ESPAC-3(v1)-randomised studies. ${ }^{14}$ Clinical and pathological characteristics of the original patient populations have been described earlier. ${ }^{3,4,6,11}$ A summary of the original trial populations and outcomes are displayed in Table 1.

Scoring and Cox PH regression analyses of DCTD and RRM1 Representative images of scoring grades 0 (negative), 1 (weak), 2 (moderate), and 3 (strong) of DCTD and RRM1 are displayed in Fig. $1 \mathrm{a}-\mathrm{h}$, and the distribution of scores in Supplementary Table 1. Cox $\mathrm{PH}$ regression analysis did not reveal any significant association with overall survival of mean DCTD expression level in the 5-fluorouracil with folinic acid group (HR 1.15, $p=0.33$ ), gemcitabine group (HR 0.93, $p=0.65$ ), or the observational group (HR 1.14, $p=0.64$ ). Analysis of mean RRM1 expression levels also did not reveal any significant association with overall survival in the 5-fluorouracil with folinic acid arm (HR 1.14, $p=0.42$ ), the gemcitabine arm (HR 0.96, $p=0.79$ ) or the observational 
subgroup (HR 1.97, $p=0.20$ ). Since univariate regression analysis did not reveal any significant association with overall survival for either DCTD or RRM1 expression, further multivariate analyses for other prognostic markers were not performed.

Median overall survival and log-rank tests of DCTD-low vs. DCTDhigh expression and RRM-low vs. RRM1-high expression Patients were grouped according to DCTD and RRM1 expression into low (scoring 0-1) and high (scoring 2-3) expression (Kaplan-Meier curves in Fig. 2a-d). Log-rank testing did not reveal any significant differences in any of the treatment arms $\left(X^{2}{ }_{\mathrm{LD}} p\right.$ values given in Fig. $2 a-d)$. An alternative splitting was performed, where patients were categorised as negative $($ score $=0)$ vs. positive (scores 1-3) expression, without revealing any significant differences in any of the studied subgroups (data not shown). For the observation subgroup, Kaplan-Meier curves and log-rank testing were not performed due to the low number of patients in the respective stratum.

\section{DISCUSSION}

Intratumoural DCTD and RRM1 protein expression was analysed in patient samples from the ESPAC-3(v2) trial with patients randomised to either gemcitabine or 5-fluorourcal with folinic acid $^{4}$ following pancreatic resection, and also in patients from the ESPAC-1 and ESPAC-3(v1) trials not receiving post-operative chemotherapy. None of the analysed biomarkers were associated with overall survival. To the best of our knowledge, we are the first group to publish data on DCTD protein expression in pancreatic cancer specimens. In line with our results, Ashida et al. ${ }^{15}$ investigated DCTD messenger RNA (mRNA) expression in tissue samples from 35 patients with advanced pancreatic cancer before starting gemcitabine treatment, without observing any significant association with overall survival.

In a multi-centre study from France, Marechal et al. ${ }^{16}$ found that intratumoural RRM1 protein expression was not significantly associated with survival time in tissue from 208 patients with pancreatic adenocarcinoma cancer who had been given post-operative gemcitabine monotherapy. In a study from Cleveland, Xie et al. $^{17}$ found that intratumoural RRM1 mRNA expression did not have significant prognostic value in 122 patients who had had resection for pancreatic adenocarcinoma, whereas low RRM1 expression was associated with longer overall survival in the 44 patients who had received adjuvant gemcitabine. In contrast, high RRM1 expression was associated with longer overall survival in the 35 patients who had received non-gemcitabine adjuvant therapy. In a study from Japan, Nakagawa et al. ${ }^{18}$ found that RRM 1 intratumoural protein expression was an independent prognostic marker in 109 patients who had resection and post-operative gemcitabine therapy. The conflicting nature of previous reports may reflect different methodologies (mRNA and protein expression analyses, the latter being performed with different protocols and antibodies) and biases from the retrospective nature of these studies, as well as genetic heterogeneity for predictive 5fluorouracil-related toxicity. ${ }^{19}$ These biases are largely overcome by studying patients from prospective multi-centre randomised trials, as in our study. Notably, the proportion of RRM1-high in our population was comparable with the proportions of RRM1-high observed in the aforementioned studies. $^{16-18}$

In conclusion, intratumoural RRM1 and DCTD protein expression levels in patient samples from prospective randomised controlled trials involving adjuvant therapy with either gemcitabine or 5fluorouracil with folinic acid have shown no association with survival when analysed in isolation of other markers, thus by themselves they are not suitable prognostic or predictive biomarker candidates.

\section{ACKNOWLEDGEMENTS}

We are grateful to all of those who participated in, and contributed to, the ESPAC-1 and ESPAC- 3 trials and provided the tissues. We thank all of the Senior Trial Coordinators on the ESPAC trials including Charlotte Rawcliffe, Karl Harvey and Chloe Smith, and the principal Data Manager was Ronald Wall to whom we are all very grateful. We are grateful to the technical staff employed in the ESPAC-T. The trial and study were funded by Cancer Research UK: (i) stratification of adjuvant chemotherapeutic response to allow personalised choice of pyrimidine prodrug: based on levels of transporters and enzymes involved in initial metabolism. Funding record ID: 108840 (W.G.). (ii) Tissue sample collection form the ESPAC adjuvant trials in pancreatic cancer (C245/A82390) (W.G.). (iii) Trials in pancreatic cancer (ESPAC-1, ESPAC-3, Gem-Cap ESPAC QLQ). SP1984/0204 and SP2590/0101 (J.P.N.). (iv) Cancer Research UK Liverpool Cancer Trials Unit. C245/A15957 and C245/A9855 (J.P.N.). Liverpool Cancer Research UK Centre. C35628/A11588 (J.P.N.). N.O.E. was funded by Stiftelsen Onkologiska klinikernas i Linköping Forskningsfond (17 December 2013) and Region Östergötland, Sweden (LIO-418581 and LIO-338111).

\section{ADDITIONAL INFORMATION}

Supplementary information is available for this paper at https://doi.org/10.1038/ s41416-018-0005-1.

Competing interests: J.P.N. reports a consulting or advisory role for Boehringer Ingelheim Pharma GmbH \& Co. KG; Novartis Pharma AG; KAEL GemVax and Astellas. Research Funding from Taiho Pharma (Japan), PI, paid to Institution; KAEL GemVax (Korea), PI, paid to Institution; AstraZeneca, PI, paid to Institution; Pharma Nord, PI, paid to Institution. Travel expenses from NUCANA, Amgen and Mylan. B.G. reports a consulting and advisory role for Pledpharma $A B$ and Isofol AB. M.D. reports a consulting and advisory role for socar research.com and travel expenses from Imotech. M.M.L. reports consulting and advisory role for Solvay, Axcan, Abbvie, Abbott, Mylan, Nordmark, AstraZeneca, Centogene, Roche, ISIS, lonis and KMG Kliniken. Travel expenses from Falk Foundation, Roche, Abbott and Abbvie, Roche, Menarini, Abbott, Abbvie, Mylan and Nordmark. Research Funding from Deutsche Forschungsgemeinschaft (DFG), Deutsche Krebshilfe/lvlildred Scheel Stiftung, Krupp Foundation, German Federal Ministry of Education and Research (BMBF), European Union (FP7, EFRE, ESF and Horizon 20120), the State Ministy of Education and Research Mecklenburg-Vorpommern, State Minisüry of Economics MV. J.R.M. reports consulting and advisory role for Pfizer, Stock or other ownership from Pacylex Pharmaceuticals Inc. and patent or intellectual property from Pacylex Pharmaceutical Inc. J.M. reports consulting or advisory role for Metonomics Health. Research funding from AstraZeneca, patent or intellectual property from Metanomics Health, Honoraria from Boehringer Ingelheim and Falk Foundation and Travel Expenses from Falk and Celgene. J.S. reports a consulting or advisory role for Amgen. W.G. reports travel expenses from Nucana Biomed. N.C.T. reports travel expenses from Roche and Honoraria from Amgen, Roche and Shire. N.O.E. reports research funding from NUCANA. D.G. reports research funding from Celgene. D.H.P. reports research funding from Nucana Biomed and Honoraria from Bayer, Nucana Biomed, Sictex, Celgene and Baxalta. A.G.S. reports Speakers' Bureau from Amgen and Honoraria from Amgen, Sanofi, Roche, Lilly, Leo and Celgene. The remaining authors declare no competing interests.

Note: This work is published under the standard license to publish agreement. After 12 months the work will become freely available and the license terms will switch to a Creative Commons Attribution 4.0 International licence (CC BY 4.0).

\section{REFERENCES}

1. Siegel, R., Miller, K. \& Jemal, A. Cancer statistics 2017. CA Cancer J. Clin. 67, 7-30 (2017).

2. Kleeff, J. et al. Pancreatic cancer. Nat. Rev. Dis. Prim. 2, 16022 (2016).

3. Neoptolemos, J. P. et al. A randomized trial of chemoradiotherapy and chemotherapy after resection of pancreatic cancer. N. Engl. J. Med. 350, 1200-1210 (2004).

4. Neoptolemos, J. P. et al. Adjuvant chemotherapy with fluorouracil plus folinic acid vs gemcitabine following pancreatic cancer resection. JAMA 304, 1073-1081 (2010).

5. Neoptolemos, J. P. et al. Comparison of adjuvant gemcitabine and capecitabine with gemcitabine monotherapy in patients with resected pancreatic cancer (ESPAC-4): a multicentre, open-label, randomised, phase 3 trial. Lancet 389, 1011-1024 (2017).

6. Neoptolemos, J. P. et al. Adjuvant chemoradiotherapy and chemotherapy in resectable pancreatic cancer: a randomized controlled trial. Lancet 358 , 1576-1585 (2001).

7. Oettle, $\mathrm{H}$. et al. Adjuvant chemotherapy with gemcitabine and long-term outcomes among patients with resected pancreatic cancer: the CONKO-001 randomized trial. JAMA 310, 1473-1481 (2013). 
8. Oettle, H. et al. Adjuvant chemotherapy with gemcitabine vs observation in patients undergoing curative-intent resection of pancreatic cancer: a randomized controlled trial. JAMA 297, 267-277 (2007).

9. Khorana, A. A. et al. Potentially curable pancreatic cancer: American Society of Clinical Oncology Clinical Practice Guideline Update Summary. J. Oncol. Pract. 13, 388-391 (2017).

10. Costello, E., Greenhalf, W. \& Neoptolemos, J. P. New biomarkers and targets in pancreatic cancer and their application to treatment. Nat. Rev. Gastroenterol. Hepatol. 9, 435-444 (2012).

11. Greenhalf, W. et al. Pancreatic cancer hENT1 expression and survival from gemcitabine in patients from the ESPAC-3 trial. J. Natl. Cancer Inst. 106, 20-25 (2014).

12. Gilbert, J. A. et al. Cancer therapy: preclinical gemcitabine pharmacogenomics: cytidine deaminase and deoxycytidylate deaminase gene resequencing and functional genomics. Drug Metab. Dispos. 12, 1794-1804 (2006).

13. Nakano, Y. et al. Gemcitabine chemoresistance and molecular markers associated with gemcitabine transport and metabolism in human pancreatic cancer cells. $\mathrm{Br}$. J. Cancer 96, 457-463 (2007).
14. Neoptolemos, J. P. et al. Adjuvant 5-fluorouracil and folinic acid vs observation for pancreatic cancer: composite data from the ESPAC-1 and -3(v1) trials. Br. J. Cancer 100, 246-250 (2009).

15. Ashida, R. et al. Gemcitabine sensitivity-related mRNA expression in endoscopic ultrasound-guided fine-needle aspiration biopsy of unresectable pancreatic cancer. J. Exp. Clin. Cancer Res. 28, 83-83 (2009).

16. Marechal, R. et al. Levels of gemcitabine transport and metabolism proteins predict survival times of patients treated with gemcitabine for pancreatic adenocarcinoma. Gastroenterology 143, 664-674 (2012).

17. Xie, $\mathrm{H}$. et al. Predictive and prognostic roles of ribonucleotide reductase $M 1$ in resectable pancreatic adenocarcinoma. Cancer 119, 173-181 (2013).

18. Nakagawa, N. et al. Combined analysis of intratumoral human equilibrative nucleoside transporter 1 (hENT1) and ribonucleotide reductase regulatory subunit M1 (RRM1) expression is a powerful predictor of survival in patients with pancreatic carcinoma treated with adjuvant gem. Surgery 153, 565-575 (2013).

19. Matsusaka, S. \& Lenz, H. J. Pharmacogenomics of fluorouracil-based chemotherapy toxicity. Expert Opin. Metab. Toxicol. 11, 811-882 (2015). 